\title{
Surface Figure and Roughness Tolerances for NIF Optics and the Interpretation of the Gradient, P-V Wavefront and RMS Specifications
}

\author{
J. K. Lawson \\ D. M Aikens \\ R. E. English, Jr. \\ W. T. Whistler \\ W. House \\ M. A. Nichols
}

This paper was prepared for submittal to the 44th Annual Meeting of the International Symposium on Optical Science, Engineering, and Instrumentation

Denver, Colorado

July 18-23, 1999

July 1999

This is a preprint of a paper intended for publication in a journal or proceedings. Since changes may be made before publication, this preprint is made available with the understanding that it will not be cited or reproduced without the permission of the author. 


\section{DISCLAIMER}

This document was prepared as an account of work sponsored by an agency of the United States Government. Neither the United States Government nor the University of California nor any of their employees, makes any warranty, express or implied, or assumes any legal liability or responsibility for the accuracy, completeness, or usefulness of any information, apparatus, product, or process

disclosed, or represents that its use would not infringe privately owned rights. Reference herein to any specific commercial product, process, or service by trade name, trademark, manufacturer, or otherwise, does not necessarily constitute or imply its endorsement, recommendation, or favoring by the United States Government or the University of California. The views and opinions of authors expressed herein do not necessarily state or reflect those of the United States Government or the University of California, and shall not be used for advertising or product endorsement purposes. 


\title{
Surface figure and roughness tolerances for NIF optics and the interpretation of the gradient, $P-V$ wavefront and RMS specifications
}

\author{
J.K. Lawson, D.M.Aikens, R.E. English, Jr.,W.T. Whistler, \\ W. House, M.A. Nichols \\ University of California \\ Lawrence Livermore National Laboratory \\ Livermore, CA 94551
}

\begin{abstract}
In a high energy laser system such as the National Ignition Facility (NIF), the ability to focus light into as small a spot as possible at the highest possible fluence is highly dependent on the quality of the optics used in the system. Typically, surface form errors and transmitted and reflected wavefront errors are specified in terms of a peak-to-valley wavefront error (P-V), or occasionally in terms of an RMS wavefront error (RMS) ${ }^{1}$. It has been shown, however, that the parameter that most closely correlates with beam focusability is neither of these, but the RMS of the gradients of the wavefront error (RMS Gradient) ${ }^{2}$. Further, the spatial frequency of the wavefront error plays a significant role in the way that a given error effects the performance of the laser system, so careful attention must be paid to how the spatial filtering is both specified and accomplished. Since ISO 10110 has no specific provisions for a gradient specification, LLNL has developed its own notation and procedures for these critical specifications.
\end{abstract}

In evaluating surface figure errors as specified by the NIF drawings, modern phase modulating interferometers (PMI) will be used. In addition to performing QA testing of the optics, LLNL intends to utilize the software capabilities of the instruments to obtain the information to model the wavefront of the 131 passes through various optical elements comprising the NIF "front end" ". A typical transmitted wavefront error call-out for an optic in the front end is:

$\lambda / 8$ P-V, $\lambda / 40$ RMS, $\lambda / 30 /$ cm RMS gradient,

with these values evaluated for spatial periods $>2 \mathrm{~mm}$ only.

Tests will be performed and documented after coating and as installed in the specified mechanical mounts. This paper describes the evaluation of the wavefront error for NIF small optics including specifications over a given spatial period callout, the proper low pass filtering of the data and the allowable filtering and settings that can be applied to obtain proper wavefront data.

This paper also describes the origin and evolution of other NIF wavefront and roughness specifications, and gives examples. Since the wavefront requirements and hence the specifications vary for the different systems in the NIF, we will focus on one system, the injection laser system (ILS) or "front end". Also discussed will be the metrology and data manipulation requirements for the large aperture optics. Finally, clarification will be given to the differences between various versions of the RMS wavefront and roughness specifications allowed in ISO 10110, and how they contrast to the RMS roughness specifications used in ANSI-Y14.5.

Keywords: P-V wavefront, RMS measurements, gradient NIF, optics, ISO10110, specifications, surface figure, RMS, gradient, wavefront 


\section{INJECTION LASER SYSTEM PERFORMANCE REQUIREMENTS}

The main NIF laser is comprised of 192 beamlines. The pulses that seed these beamlines will be generated by a total of 48 ILS assemblies. The performance requirements for an individual ILS that are consistent with NIF total performance requirements have been previously outlined ${ }^{4}$ and are summarized in Table 1 . These requirements were derived from the laser output requirements and are consistent with both propagation and lens design models for the ILS. We have used these requirements as the basis for the optics specifications.

\section{OPTICS SPECIFICATIONS}

\subsection{RMS Gradient}

In order to meet the ILS divergence budget, we have derived the rms gradient requirements consistent with a given divergence ${ }^{5}$. A reasonable assumption regarding the wavefront slopes of the a wavefront after passing through an optical train is that the final slope values are the result of the uncorrelated addition of the slope errors associated with each individual optical component. That is, we can write

$$
\begin{aligned}
& \alpha_{x, \text { final }}(x, y)=\sum_{i=1}^{N_{e}} \alpha_{x, i}(x, y), \\
& \alpha_{y, \text { final }}(x, y)=\sum_{i=1}^{N_{e}} \alpha_{y, i}(x, y),
\end{aligned}
$$

where $N_{e}$ is the total number of optical elements in the beam path. Given our assumption of uncorrelated addition, and equal $x$ and $y$ slope variances for individual elements, we find that the final $x$ and $y$ slope variances are given by

$$
\begin{aligned}
& \sigma_{\alpha_{x}, \text { final }}^{2}=N_{e} \sigma_{x y}^{2}, \\
& \sigma_{\alpha_{y}, \text { final }}^{2}=N_{e} \sigma_{x y}^{2} .
\end{aligned}
$$

A direct implication of Eq. 1 is that the final wavefront $x$ and $y$ slopes $\alpha_{x, f i n a l}(x, y)$ and $\alpha_{y, \text { final }}(x, y)$ are also Gaussian random variables with zero means and the variances as given in Eq. 2. Following the same analysis as in the previous section, we can then write the distribution of the final wavefront slope magnitudes as

$$
f_{|\alpha|_{\text {final }}}\left(|\alpha|_{\text {final }}\right)=\frac{|\alpha|_{\text {final }}}{N_{e} \sigma_{x y}^{2}} \exp \left(\frac{-|\alpha|_{\text {final }}^{2}}{2 N_{e} \sigma_{x y}^{2}}\right) \text {. }
$$

Now let us define a specification for $|\alpha|_{\text {final }}$ such that $80 \%$ of all slope magnitude values over the final wavefront fall within the total divergence budget $\Omega$. The probability that the final wavefront slope magnitude at point $(x, y)$ is less than or equal to $\Omega$ is given by

$$
\operatorname{Prob}\left\{|\alpha(x, y)|_{\text {final }} \leq \Omega\right\}=\int_{0}^{\Omega} f_{|\alpha|_{\text {final }}}\left(|\alpha|_{\text {final }}\right) d|\alpha|_{\text {final }}
$$

and so we then require that

$$
\frac{1}{N_{e} \sigma_{x y}^{2}} \int_{0}^{\Omega}|\alpha|_{\text {final }} \exp \left(\frac{-|\alpha|_{\text {final }}^{2}}{2 N_{e} \sigma_{x y}^{2}}\right) d|\alpha|_{\text {final }} \geq 0.80
$$


Solving this integral yields

$$
1-\exp \left(\frac{-\Omega^{2}}{2 N_{e} \sigma_{x y}^{2}}\right) \geq 0.80
$$

which finally reduces to

$$
\sigma_{x y} \leq \frac{\Omega}{\sqrt{-2 N_{e} \ln (0.2)}}
$$

From Table 1, the total system divergence requirement for the ILS can be estimated from the $80 \%$ spot size requirement, i.e. $\Omega \sim 6.5$ microradians. We would like to allocate half of that amount to the fabrication of generic optics, reserving the remainder for rods and rotators (which are expected to have large gradients due to inhomogeneities) and alignment errors. Thus, assuming the two contributions will add as the root of the sum of the squares, $\Omega=4.6$ microradians. However, this requirement is upon injection into the main laser cavity. The magnification difference between the main cavity and the ILS increases the ILS divergence approximately tenfold. Thus, for our estimate, $\Omega \sim 46$ microradians.

Accounting for both correlated and uncorrelated passes, the number of effective optical passes in the ILS is roughly $\mathrm{Ne}=200$. Plugging values of $\Omega=46$ microradians and $\mathrm{Ne}_{\mathrm{e}}=200$ for the NIF front-end design requirements and configuration into Equation 7, we obtain the following as specifications for the transmitted wavefront slopes measured for individual small optical components in the NIF (expressed in waves at $633 \mathrm{~nm}$ ):

$$
x \text { and } y \text { wavefront slope rms values } \sigma_{x y} \leq \frac{\lambda / 35}{\mathrm{~cm}}
$$

It can be shown that the rms of the slope magnitudes (taken about zero) is related to $\sigma_{\mathrm{xy}}$ by

$$
\begin{aligned}
\text { rms slope magnitude } & =\sqrt{2} \sigma_{x y} \\
& \leq \frac{\lambda / 25}{\mathrm{~cm}}
\end{aligned}
$$

We have chosen to tighten this specification slightly to allow for design margin, requiring an $\mathrm{rms}$ gradient of $\lambda / 30 / \mathrm{cm}$. (Note that the terms slope magnitude, gradient and gradient magnitude are often used interchangeably.) Due to the magnification difference of the ILS relative to the main NIF laser chain, the rms gradient is taken over spatial frequencies up to approximately $0.5 \mathrm{~mm}^{-1}$ (i.e., spatial periods of greater than $2 \mathrm{~mm}$ ).

\subsection{P-V and RMS}

Referring back again to Table 1, the ILS wavefront error expressed in terms up to third order must be less than 1.5 waves. This establishes the basis for the ILS system P-V and rms wavefront specifications. Taking the optical configuration, accounting for coherent and incoherent passes, and assuming a ratio of 5:1 between P-V and rms, we arrive at a detailed budget allotment for the various ILS optics. For a typical optics, these values are $\lambda / 8 \mathrm{P}-\mathrm{V}$ and $\lambda / 40 \mathrm{RMS}$. For several of the optics systems, we are relying on our CODE-V design for specifications of maximum power and irregularity allowed on the surfaces. These specifications will be depicted using the ISO-10110 notation for surface form, i.e., 3/. The following callout is an example of a surface specification that allows a third of a fringe of focus and an eighth of a fringe of surface irregularity:

$$
3 / 0.3(0.125) \text {. }
$$


Note that we have departed from strict ISO-10110 notation in two ways. 1) We use $632.8 \mathrm{~nm}$ as the base wavelength in the conversion of fringes and waves by stating the reference wavelength in the drawing notes. 2) We use the American convention of decimal representation instead of the European convention using commas.

In other cases, surfave quality is not the primary requirement, but transmitted wavefront is critical. For these cases, surface P$\mathrm{V}$ and rms specifications are replaced by a transmitted wavefront specification. The transmitted wavefront quality is called out in the notes since there is not currently a specific ISO-10110 notation for transmitted wavefront quality.

\subsection{Surface Roughness}

Surface roughness will be treated for two different cases for the front end optics. One case involves the high frequency behavior of the wavefront. Evaluated for spatial periods shorter than $2 \mathrm{~mm}$, an rms roughness will be specified in the notes of the drawing. This specification will generally read "less than $0.8 \mathrm{~nm}$ ". The second case is edge finish on the optics. In both cases, we use the ISO 10110 notation for surface texture. The "check mark" notation is similar in appearance to the ASMEY14.5 callout for surface texture, but differs in the allowed symbols to denoted type of finish. G represents a ground or matte finish while $\mathrm{P}$ represents a polished one. $\mathrm{Rq}$ specifies the rms roughness in microns.

\section{SPECIFICATION VERIFICATION: SPECIAL CONSIDERATIONS}

\section{1. $\quad$ Spatial Filtering}

The majority of optical wavefront specifications for NIF are derived either from their impact on the output of the laser system or from risk of optical damage at high power operation. Because the fluences present in the ILS are sufficiently low that nonlinear processes and associated damage are not generally an issue in that section of the laser chain, specifications are most critical over those spatial frequencies that will be transmitted to the main laser chain. In the main laser chain, rms gradient specifications help control the focusability of the output beam. The rms gradient for the main laser chain is defined for spatial scalelengths up to $33 \mathrm{~mm}^{6}$. Due to the difference in beam size between the ILS and the main laser chain, this becomes a specification for spatial scale lenghts up to $3.3 \mathrm{~mm}$ in the power amplification module (PAM) and input sensor package (IPS) and up to $4.0 \mathrm{~mm}$ in power amplifier beam splitter telescope (PABST). Currently, we are specifying over a single, slightly broader spatial wavelenth range for convenience and to allow some margin for design modifications. We have defined the spatial wavelenght cuttoff at $2 \mathrm{~mm}$ for all optics in the ILS.

Since the rms gradient is specified over a range of spatial scalelengths, spatial filtering plays a central role in the specification verification. Unfortunately, there exist some concerns over how commercial intereferometry software handles spatial filtering. If care is not taken to minimize the effects of Gibbs modulation ${ }^{7}$, filtered interferograms can display more modulation than the original data. The effect of finite sampling must also be mitigated by windowing or other techniques to minimize the artifacts that sometimes accompany spatial filtering. Lastly, the way drop-outs and no-data regions are handled can also effect the results. For these reasons, we have created post-processing routines to test the effect of commercial software algorithms on the values calculated for the the rms gradient and power spectral density (PSD). Since the PSD is highly sensitive to the nature of the algorithm used and is a critical specification for the large aperture optics, we have decided to use custom post-processing software to verify compliance of NIF large aperture parts with the wavefront specifications. In the case of small optics, however, we have instead decided to use commercial software capabilities with specific instructions on the way the specifications are to be verified. Lowpass spatial filtering of the interferogram with a cutoff wavelength of $2 \mathrm{~mm}$ will be done before computation of the rms gradient. The filtering algorithm used must be demonstrated to produce no artifacts that would alter the calculation of the rms gradient. We hope that the necessary improvements to the filtering and analysis algorithms in the interferometry software will be implemented in the near future.

\subsection{Measurements of NIF-type Optics}

Specifications, regardless of the method by which they were developed, are pointless unless parts can be manufactured to meet them. Thus, we have set rms gradient specifications not only to meet performance goals, but also to be consistent with real optics. We measured several "representative" optics for the ILS and calculated where they would meet the current specifications. A small subset is shown in Table 2. In general, the optics that we have measured that are "typical" of an ILS 
optics have rms gradients in the range from $\lambda / 30 / \mathrm{cm}$ to $\lambda / 50 / \mathrm{cm}$. The notable exceptions are rods, rotators and waveplates which have bulk properties that influence the gradient measurement.

Figures 1 and 2 depicts different stages of the verification calculations and illustrates the effect of spatial filtering on the value of the gradients. We have verified that the unique, "worm-like" pattern seen in the gradient is associated with the optic and not the interferometer optics or air turbulence. The pattern replicated on sequential measurements and followed the optic as a series of measurements were taken of the optic rotated at multiple of 90 degrees. The structure visible has a characteristic size that is associated with the cutoff frequency of the low pass filter.

\section{CONCLUSION}

The optics in the ILS play an important role in the NIF. Not only do they provide the energy required to feed the many beamlines, but they provide the initial contribution to the final wavefront error. As such, their specifications reflect the impact of that contribution. We specifiy the wavefront error only over the spatial wavelengths that will play a role in the final output. We use the rms gradient as the primary specification to control the detrimental effects of wavefront error on the final spot size and have specific procedures that outline how it is calculated.

In addition to this non-traditional application of optical specifications, we have a host of traditional optical specifications that result from our optical design. Surface form and roughness are chief among these specifications. We have chosen to depict them on our drawings using ISO 10110 notation with some modifications.

Lastly, we have measured prototypic ILS optics to convince ourselves (and others) that our specifications are reasonable. These results are encouraging. The measurements also form the beginnings of a database of interferograms upon which we are drawing for propagation modeling of the ILS.

\section{ACKNOWLEDGEMENTS}

This work has been conducted in support of the optical design effort for the National Ignition Facility at the Lawrence Livermore National Laboratory. This work was performed under the auspices of the U.S. Department of Energy by the Lawrence Livermore National Laboratory under contract No. W-7405-ENG-48.

\section{REFERENCES}

1. D. M. Aikens, "The origin and evolution of the optics specification for the National Ignition Facility", Optical Manufacturing and Testing, Proc. SPIE Vol. 2536, pp. 2-12, 1995.

2. J. K. Lawson, et.al., "NIF Optical Specifications - The Importance of the RMS Gradient Specification", Proceedings of the Solid State Lasers for Applications to Inertial Confinement Fusion Third Annual Conference, Monterey, California, June 7-12, 1998, to be published.

3. D. M. Aikens, "National Ignition Facility small optics overview", Optical Manufacturing and Testing III, SPIE International Conference on Optical Science Engineering and Instrumentation, Denver, Colorada, July 18-23,1999, to be published.

4. Jerome Auerbach, et al., "NIF Laser System Performance Ratings", supplement to SPIE Vol.3492, 1998.

5. John Downie, "Specifications of finished optical components at low spatial frequencies", NIF Design Document \#NIF0000325 , July 25, 1996.

6. J.K. Lawson, D.M. Aikens, R.E. English, Jr., C.R. Wolfe, "Power spectral density specifications for high-power laser systems", Specification, Production and Testing of Optical Components and Systems, SPIE Proc. Vol. 2775, pp-345-356 (1996).

7. See for example, Electromagnetic Fields and Waves, $3^{\text {rd }}$ Edition, Paul Lorrain, Dale Corson and Francois Lorrain, (W.H. Freeman and Co, New York, 1988), p221. 
Flowdown from the doubler to the injection laser system of the primary criteria and functional requirements for ignition pulses*: 192 beams

Per beam rating with $1 \AA \AA$ bandwidth undispersed

\begin{tabular}{|l|c|c|}
\hline \multirow{2}{*}{ Energy* } & Indirect drive & Direct drive \\
\hline Peak Power & $0.88 \mathrm{~J}$ & $0.35 \mathrm{~J}$ \\
\hline Near feld contrast & $344 \mathrm{MW}$ & $186 \mathrm{MW}$ \\
\hline Focal spot & $7.5 \%$ & $7.5 \%$ \\
Radii & $95 \%: 13.4 \mu \mathrm{rad}$ & $95 \%: 13.5 \mu \mathrm{rad}$ \\
& $80 \%: 6.5 \mu \mathrm{rad}$ & $80 \%: 6.5 \mu \mathrm{rad}$ \\
\hline Prepulse & $50 \%: 2.4 \mu \mathrm{rad}$ & $50 \%: 2.4 \mu \mathrm{rad}$ \\
\hline Tempor al puls ${ }^{\star}$ & $5 \mathrm{~kW}$ & $5 \mathrm{~kW}$ \\
dynamic range & $\sim 95: 1$ & $\sim 79: 1$ \\
\hline Tempor al shape & Continuous & Continuous \\
\hline Rms deviation in & $3 \%$ over any & $3 \%$ over any \\
en ergy or power & $2 \mathrm{~ns}$ interval & $2 \mathrm{~ns}$ interval \\
betwe en quads & & 12.5 \\
\hline Pulse ength (nsec) & 21 & - \\
\hline Beam positioning & - & \\
\hline
\end{tabular}

Rated with $3 \AA$ A bandwidth undispersed

Table 1. Performance requirements for the ILS derived from

NIF primary criteria and functional requirements

\begin{tabular}{|l|l|l|l|l|l|l|}
\hline optic & filename & $\begin{array}{l}\text { TWF/ } \\
\text { RWF }\end{array}$ & $\begin{array}{l}\text { P-V } \\
\text { (waves) }\end{array}$ & $\begin{array}{l}\text { Rms } \\
\text { (waves) }\end{array}$ & $\begin{array}{l}\text { Power } \\
\text { (waves) }\end{array}$ & $\begin{array}{l}\text { rms gradient } \\
\text { (waves/cm) }\end{array}$ \\
\hline Unclad rod & $990507 \mathrm{~b}$ & TWF & .198 & .036 & -0.124 & $0.051^{*}$ \\
\hline rotator & Frtrans1 & TWF & .188 & .037 & -0.147 & $0.063^{*}$ \\
\hline ISP mirror & $990406 \mathrm{a}$ & RWF & .058 & 0.11 & & 0.023 \\
\hline half-waveplate & $990322 \mathrm{a}$ & TWF & .167 & .034 & & 0.062 \\
\hline polarizer & $990112 \mathrm{ab}$ & TWF & 0.087 & 0.020 & & 0.027 \\
\hline
\end{tabular}

* after subtraction of power 

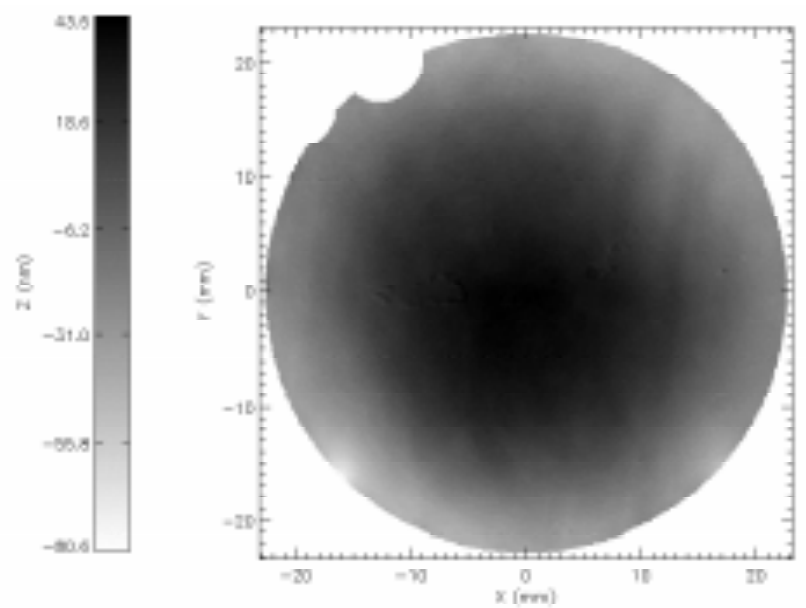

(a)
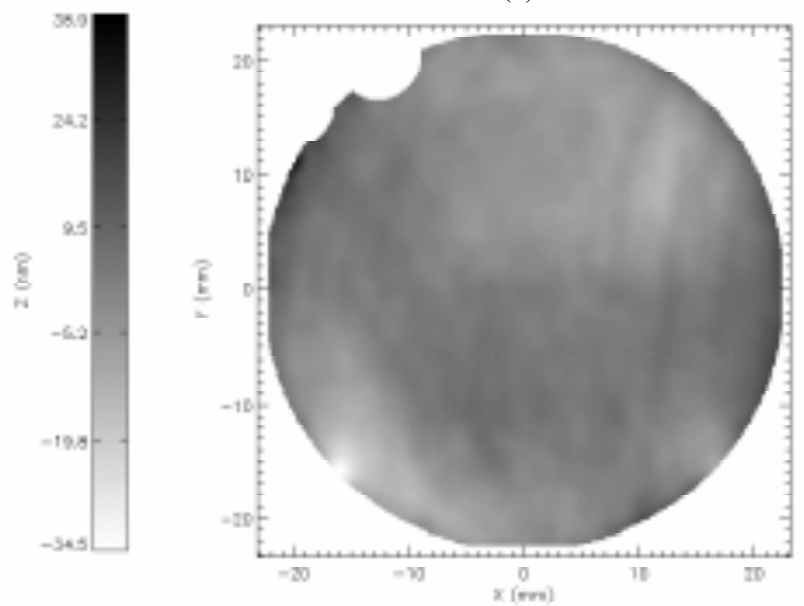

(b)
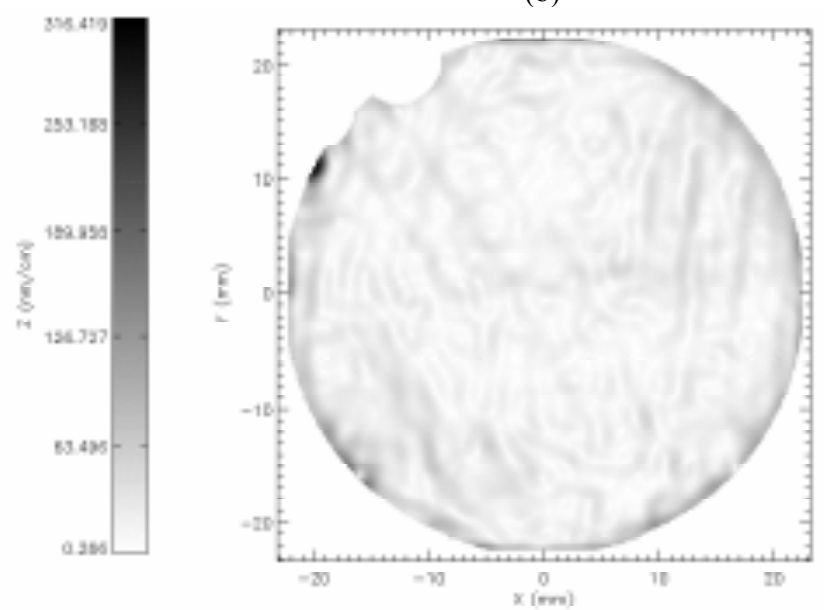

(c)

Figure 1. Various stages of analysis of a measurement of a laser rod taken by a phase modulating interferometer (PMI). (a) is the original phase measurement. (b) is (a) after removing focus and applying a Fourier low-pass filter with a $2 \mathrm{~mm}$ cutoff. (c) is the gradient of (b). 

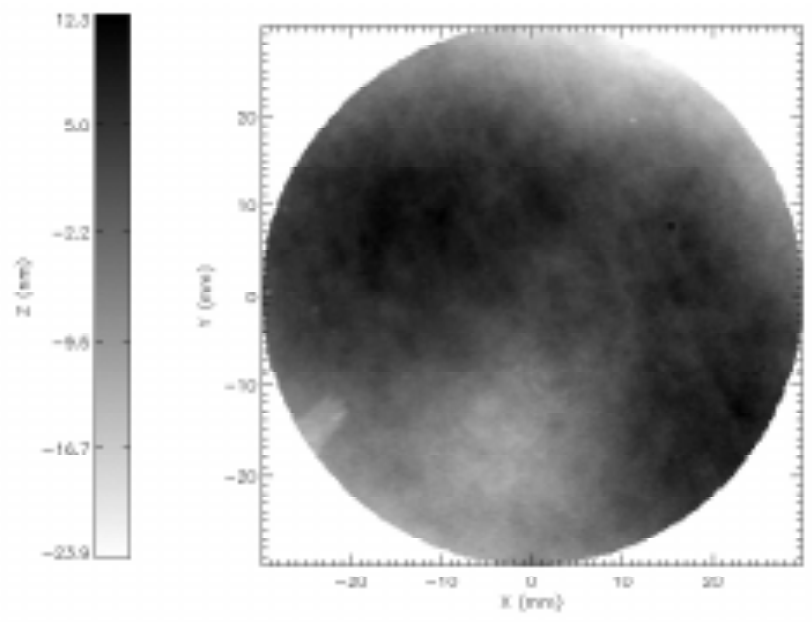

(a)
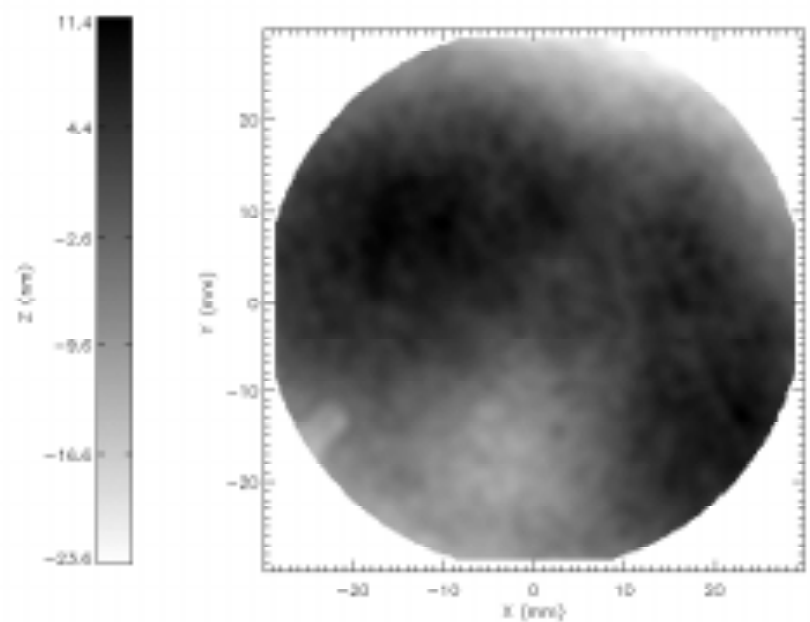

(b)
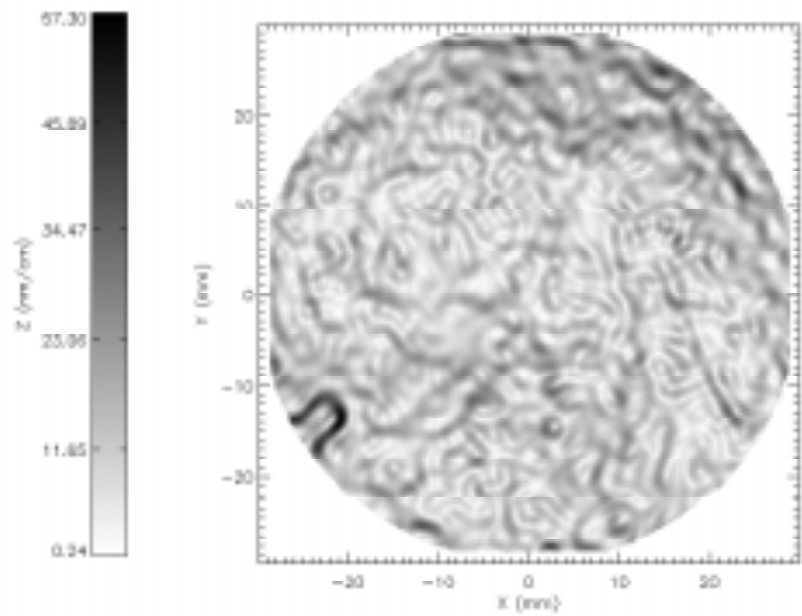

(c)
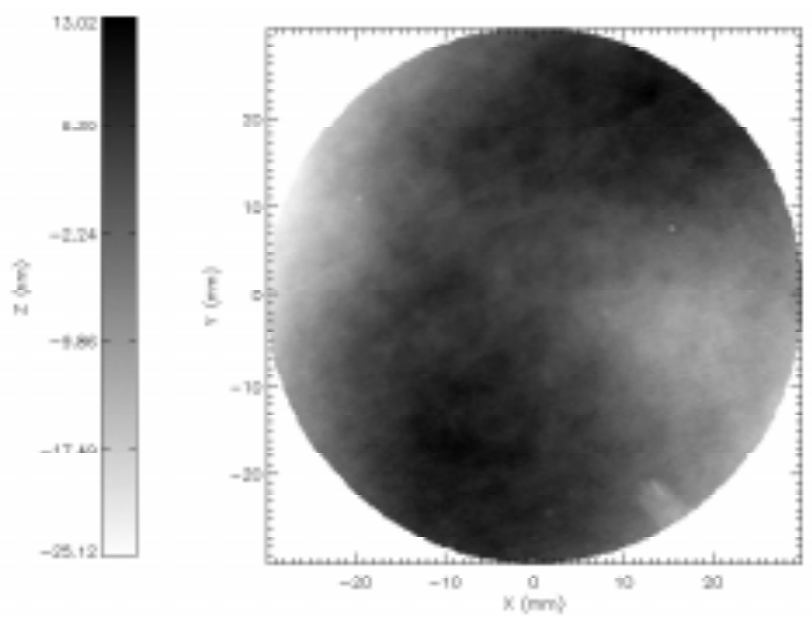

(d)
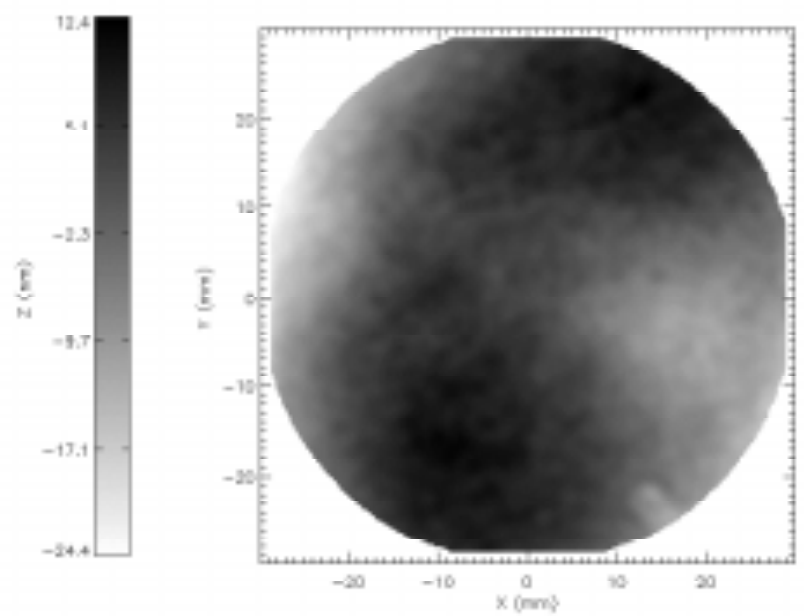

(e)
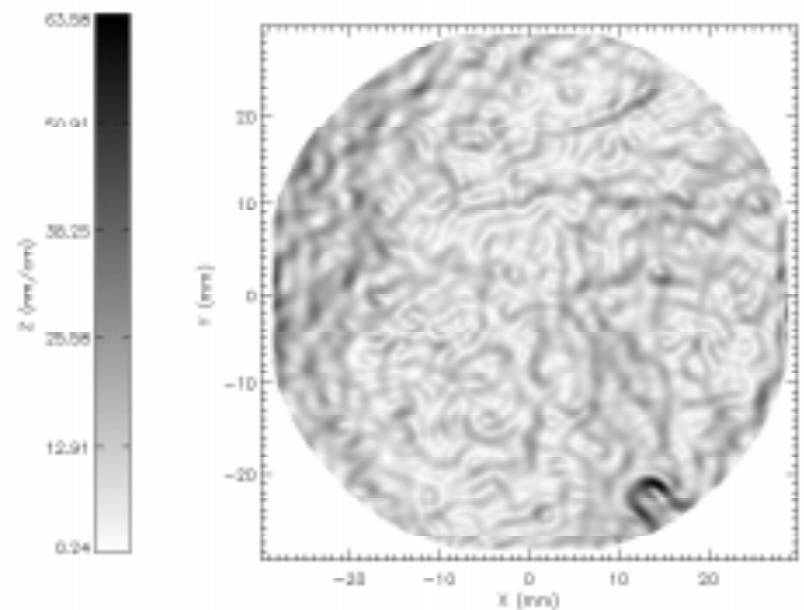

(f)

Figure 2. Various stages of analysis of a measurement of a mirror taken by a phase modulating interferometer (PMI). (a) is the original phase measurement. (b) is (a) after a Fourier low-pass filter with a $2 \mathrm{~mm}$ cutoff. (c) is the gradient of (b). Images (d) - (e) represent the same sequence with the mirror rotated by $90^{\circ}$ counterclockwise. The unusual pattern seen in the gradient calculation is characteristic of the filtered phase and rotates with the mirror. The structure seen in the lower left of (a) is a reference mark added to the mirror. 